\title{
The Comparison of the DASH, Hypocaloric, Mediterranean/Low Glycemic Diet/Low Carbohydrate, as a Nutritional Intervention in Polycystic Ovary Syndrome in Overweight Women: A Systematic Review
}

\author{
Stephanie Le (Corresponding author) \\ Health and Human Performance Department, University of Houston, Houston, Texas \\ E-mail: Stle7@uh.edu \\ Kevin Haubrick \\ Health and Human Performance Department, University of Houston, Houston Texas \\ E-mail: khaubrick@uh.edu
}

Received: May 6, 2021 Accepted: July 10, $2021 \quad$ Published: July 17, 2021

doi:10.5296/jfs.v10i1.18609 URL: https://doi.org/10.5296/jfs.v10i1.18609

\begin{abstract}
There is substantial evidence supporting individuals with polycystic ovary syndrome (PCOS) benefit from lifestyle changes through a nutrition intervention that improves small to moderate weight loss, restore ovulation, improve menstrual regularity, along with clinical (anthropometrics) and biochemical features (glucose tolerance, insulin, menstrual cycle, testosterone levels, free androgen index (FAI), and sex hormone binding globin). The objective of this systematic review was to evaluate individuals with PCOS and their body responses to different diets following a DASH, Mediterranean/Low-glycemic diet/low carbohydrate diet, and hypocaloric, diet in the improvement of clinical (anthropometrics) and biochemical (glucose tolerance, insulin, menstrual cycle, testosterone levels, free androgen index [FAI], and sex hormone binding globin) features. The literature was analyzed in different diet groups supporting nutrition intervention for PCOS in overweight/obese women in term of finding in clinical (anthropometrics- body mass index [BMI], waist circumference, and weight loss) and biochemical features (glucose tolerance, insulin, menstrual cycle,
\end{abstract}


testosterone levels, FAI, and sex hormone binding globin), which will provide evidence to determine the best nutrition intervention for PCOS. This systematic review highlighted significant improvements in BMI, insulin resistance, menstrual irregularity and decrease testosterone levels in PCOS patients when following different diets (DASH, hypocaloric, Mediterranean/low-glycemic diet/low carbohydrate diet) when they are being compared among each other. Each diet supported different improvements in anthropometrics or biochemical biomarkers.

Keywords: DASH, Mediterranean, Hypocaloric/ Calorie-restricted, Low Glycemic/ Low Carbohydrate / Gluten Free, Dietary Intervention, Polycystic Ovary Syndrome, Insulin Resistance, Glucose Tolerance

\section{Introduction}

Polycystic Ovary Syndrome (PCOS) is the most common endocrine disease characterized by a combination of signs and symptoms of androgen excess (testosterone) and ovarian dysfunction (reproductive hormones) (Escobar, 2018). PCOS is frequently associated with insulin resistance, type II diabetes, metabolic disorders, excessive weight or obesity, and cardiovascular risk factors; however, the most significant clinical indicator of PCOS is hyperandrogenism (Zeng et al., 2020). Polycystic Ovary Syndrome (PCOS) is a disorder involving irregular menstrual cycles and often produced excess male hormones like androgen levels. The study on how food directly affect PCOS is hypothetically questioned; therefore, this systematic review will investigate how differing diets adversely affect PCOS or suggest potentially new nutrition therapy for PCOS by reviewing clinical and biochemical features along with reproductive hormones and menstrual regularity.

Women with PCOS have excess androgen levels along with insulin resistance and hormone imbalance of estrogen levels, which can result in increased biomarkers of oxidative stress and characteristics of lipids. PCOS has shown to be associated with type 2 diabetes mellitus (T2DM), ovarian cancers, cardiovascular diseases and increased risk for infertility (Teede et al., 2013; Rasool et al., 2019). The initial treatment for PCOS includes incorporating more physical activity, and lifestyles change such as weight management (Kazemi et al., 2018). A weight loss of 5\%-10\% can show improvement in hyperandrogenic, reproductive, and metabolic features (Jiskoot et al., 2017). The most current treatment is using oral contraceptive pills and relying on metformin to help stimulate regular menstrual cycle and reduce hyperinsulinemia and ovarian androgens productions in women (Asemi et al., 2014; Aicher et al., 2012). Le Donne and colleagues noted weight loss was dependent on adding metformin to diet and myoinositol was effective in restoring menstrual cycle. Further investigation is needed to confirm metformin and myoinositol on improvement of body composition (LeDonne et al., 2012). The first line of treatment for PCOS includes lifestyle changes and dietary management as weight loss lead by modulating your dietary intake (Asemi et al., 2014; Jiskoot et al., 2017; Ravn et al., 2013), which seems to improve hormone parameters (Rajaeieh et al., 2013). Therefore, this systematic review will provide or suggest new nutrition therapy by evaluating individual's responses following different diet (DASH, Mediterranean, Hypocaloric, Ketogenic, and Low Dairy/ Gluten Free) and highlighted improvement on any anthropometrics (BMI, waist circumference, and weight loss) and biochemical features (glucose tolerance, insulin, menstrual cycle, testosterone levels, free 
androgen index (FAI), and sex hormone binding globin).

\section{Methods/ Literature Search Strategy}

A literature search was conducted on peer reviewed research trials registered on PubMed and University of Houston MD Anderson Library from 2011-2021 utilizing the search term: PCOS-Polycystic Ovary Syndrome AND Dietary Intervention AND Insulin Resistance, OR PCOS Diet Intervention, OR Hormones Imbalance in Overweight and Obese Women with PCOS. Studies included in this review were all primary research: clinical trials/ randomized controlled trials. Secondary research articles (systematic review, meta-analysis, etc.) were excluded. Filters were added to show only English, peer reviewed journal articles, full text only, and publication date within 10 years. Studies on animals were excluded and only human studies were included. Articles were screened and excluded if not original research and did not include participants with Polycystic Ovary Syndrome and did not use any nutrition intervention. The Preferred Reporting Items for Systematic Review and Meta-Analyses (PRISMA-P) flow diagram (Moher et el., 2015) was used to complete the systematic review.

\section{Pathophysiology of Polycystic Ovary Syndrome}

The exact pathophysiological mechanism leading to PCOS phenotype remains unknown. Researchers explain the syndrome as an intrinsic ovarian disorder (excess ovarian production of androgens, hypothalamic-pituitary dysfunction (increase secretion of luteinizing hormone), and metabolic disorder being insulin resistance (defined as reduced glucose response to a given amount of insulin), accompanied by compensatory hyperinsulinemia (Costello et al., 2007). Hyperinsulinemia suppresses sex hormone binding globin (SHBG) and stimulates both ovarian and adrenal androgen secretion leading to premature follicular atresia and anovulation (Costello et al., 2007), which is define as absence of ovulation- release of an egg. Traditionally, first line treatment for women who do not desire pregnancy is an oral hormone contraceptive agent (OCPs) containing estrogen and progestin (Mayhew, 2011). The contraceptive treats excess androgenism and menstrual irregularity. The first line of treatment for individuals who desires pregnancy includes Metformin and Clomiphene (Mayhew., 2011). The combination of Metformin and Clomiphene is more effective than either alone (Mathur et al., 2008). Clomiphene is a nonsteroidal ovulation-induction medication, while Metformin inhibits ovarian gluconeogenesis causes reduction in ovarian androgen production (Mayhew., 2011). Metformin has many benefits for patients with PCOS; many of the few are: improve fertility, improve insulin resistance to prevent long term risk of type 2 diabetes, assist with weight loss and decrease testosterone while improving hirsutism (Mayhew., 2011).

While medications can help relieve signs and symptoms of PCOS, dietary intake can help improve insulin resistance, manage weight loss, and other clinical (anthropometrics) and biochemical features (free androgen index (FAI), glucose tolerance, insulin, and testosterone levels) along with lifestyle modifications with increased physical activity (Costello et al., 2012). The search process (Figure 1) was performed on online databases (PubMed and University of Houston MD Anderson Library) and featured the aforementioned inclusion/exclusion criteria. To search for articles addressing dietary intervention for PCOS patients in overweight/obese women. 


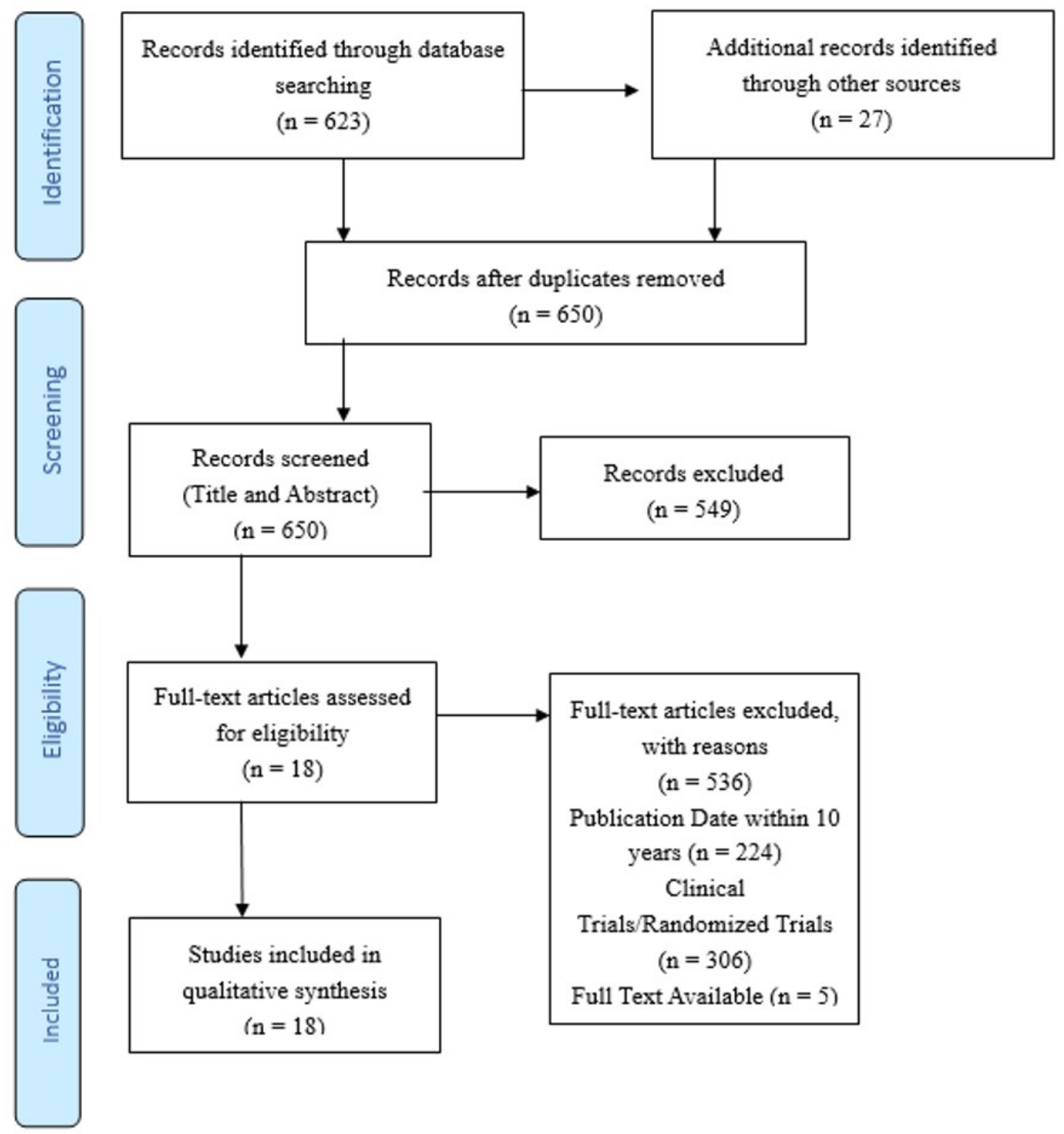

Figure 1. PRISMA flow diagram detailing database searches and article selection/inclusion

\section{Dietary Approaches to Stop Hypertension (DASH)}

Due to hyperinsulinemia, which is often associated with type 2 diabetes (caused by insulin resistance), scientists suggested the eating pattern of a Dietary Approaches to Stop Hypertension (DASH) diet can help with the management of diabetes. The dietary approach is balanced, nutritious, and maintainable eating pattern that can improve many health factors, including insulin resistance, overweight/obese, hyperlipemia, and hypertension (Campbell, 2017). The DASH diet is known for lowering blood pressure; however, its beneficial effects have also been reported in type 2 diabetes (T2DM) and metabolic syndrome. According to the 2012 Standards of Medical Care in Diabetes (which are updated every 10 years), the DASH diet lifestyle therapy consists of reducing sodium intake ( $<1500 \mathrm{mg}$ per day), increase 


\section{Macrothink}

fruits and vegetables (8-10 servings a day), lower fat dairy products (2-3 serving per day), and avoiding excessive alcohol consumption while increasing physical activity levels may also positively affect glycemia and lipid control (Standards of Medical Care in Diabetes, 2012).

Women with PCOS have excess androgen, insulin resistance, and variable amounts of estrogen exposure, all of which can result in an increase of lipid profiles and biomarkers of oxidative stress (Asemi et al., 2014). Recently, it has been suggested low carbohydrate diet, low glycemic control, and high protein diet is more beneficial influence for PCOS compared to a low fat and high carbohydrate diet (Asemi et al., 2014). Investigators also studied a low-glycemic diet in combination with medications, which has resulted in symptoms relief in patients with PCOS. Nonetheless, when scientist studied and analyzed the adherence to the recommended low carbohydrate, low glycemic diet for 12 weeks, results determined a non-significant effect on lipid profiles in women (Asemi et al., 2014). The DASH eating pattern is a low-glycemic index, low energy dense known for lowering blood pressure; however, its beneficial effects have been reported in insulin resistance, type 2 diabetes, increased inflammation, and metabolic syndrome (Asemi et al., 2014). PCOS is associated with hormonal imbalance, it is unclear if the DASH diet can affect insulin resistance and inflammation in patients with polycystic ovary syndrome. At this time lifestyle modifications that target weight reduction, insulin resistance, lipid profiles, and glycemic index is a dietary strategy appears to be beneficial for overweight/obese women with PCOS. The DASH diet has reported to improve insulin resistance, glycemic index, and lipid profiles (Azadi et al., 2016).

\subsection{Clinical Trials}

According to Campbell (2017), the DASH eating pattern is closely aligned with the United States Department of Agriculture's (USDA) dietary recommendations as outlined in the 2015-2020 Dietary Guidelines for Americans. The healthy eating pattern limits saturated fats, trans fat, added sugars, and sodium while strongly encourage the intake of vegetables, fruits, grains, oils, fat free dairy products, and a variety of proteins. The macronutrients composition outlined in Campbell (2017) eating patterns are as follows: 55\% carbohydrates, $18 \%$ protein, $27 \%$ total fat, $6 \%$ saturated fat, and $2300 \mathrm{mg}$ sodium; however, $1500 \mathrm{mg}$ sodium was tested and found to be even better for lowering blood pressure. To the knowledge of the researchers, Campbell (2017), have proven the DASH diet to be beneficial to control hypertension. Nearly two out of three with diabetes have hypertension therefore, it is reasonable to expect the eating pattern of the DASH diet would benefit from both hypertension and diabetes (Campbell., 2017). Due to proven benefits of DASH diet with patients with diabetes, a group of researchers investigate the effects of DASH eating patterns on lipid profiles, insulin resistance, androgens, body composition, weight loss, and anti-mullerian hormone in overweight/obese women with PCOS.

Asemi et al. (2014), Asemi et al. (2014), Azadi et al. (2016), and Foroozanfard et al. (2017) used a control diet and the DASH diet, both containing 50-55\% carbohydrate, $15-20 \%$ protein, and $25-30 \%$ total fats. The DASH diet was designed to be rich in fruits, vegetables, whole grains, low-fat dairy products, low refined grains, and sweets; however, the control diet is different in terms of food groups, but both were equicaloric. Table 1 shows the 
nutrition intervention in studies with participants with PCOS. Table 1 notes the number of participants in each study, the macronutrients in the control diet versus DASH diet, the study length, and the significant findings. The participants in the studies were provided dietary education by a Registered Dietitian. All study personnel and subjects were blinded to the dietary assignment to ensure bias responses, as allocation of the diet was concealed from the researchers and patients (Asemi et al., 2014); Asemi et al., 2014; Azadi et al., 2016; and Foroozanfard et al., 2017). To monitor adherence to the diet, a 3-day food record was completed throughout all studies (Asemi et al., 2014; Azadi et al., 2016; Foroozanfard et al., 2017) while Asemi et al. (2014) had participants record their dietary intake every two weeks. To control the dietary intake of the subjects throughout the study, a regular call from the Registered Dietitian was required to solve any possible problems. All study participants were given a 7-days menu cycles without food being prepare due to studies not being a feeding trial (Asemi et al., 2014; Asemi et al., 2015; Azadi et al., 2016; Foroozanfard et al., 2017). An important factor to point out was during the trials is participants were asked not to alter their routine physical activity or usual activity intake and not to consume any supplements that might influence or alter biomarkers during the intervention. This may prevent any unexpected weight loss or changes to the body.

Table 1. Description of DASH diet interventions in studies with participants with polycystic ovary syndrome

Description of DASH diet interventions in studies of participants with Polycystic Ovary Syndrome

\begin{tabular}{llll}
\hline Reference & Population & $\begin{array}{l}\text { Study } \\
\text { Length }\end{array}$ & $\begin{array}{l}\text { Authors description of } \\
\text { nutrition intervention }\end{array}$
\end{tabular}

Asemi et al. 48 overweight/ obese women (2014) diagnosed with polycystic ovary syndrome. The women were given a controlled diet $(n=24)$ or the DASH diet $(n=24)$. Both diets are calorie-restricted and contains $52 \%$ carbohydrates, $30 \%$ total fat, and $18 \%$ proteins.
8 weeks

Adherence to the DASH diet resulted in significant decrease in weight $(\mathrm{P}<$ $0.001)$, body mass index $(\mathrm{P}<$ 0.001), decreased serum insulin triglycerides and very-low density lipoprotein cholesterol levels (P interaction: 0.005). Increased concentrations of TAC and GSH (P interaction $<0.001$ and $\mathrm{P}$ interaction: 0.005 , respectively.

Asemi et al. 48 overweight /obese women 8 weeks Adherence to the DASH diet 
diagnosed with PCOS. The women were given a controlled diet $(n=24)$ or the DASH diet $(\mathrm{n}=24)$. Both diets are calorie-restricted and contains $52 \%$ carbohydrates, $30 \%$ total fat, and $18 \%$ proteins.

Azadi et al. 60 women with PCOS assigned (2016) to one of the two diets: DASH diet versus control diet. The DASH and control diets consisted of $50-55 \%$ carbohydrate, $25-30 \%$ total fat, and $\quad 15-20 \% \quad$ protein. Anthropometric, body composition, sex hormone, androgen, and testosterone were

measured before and after 3 months period.

Foroozanfard 60 overweight and obese 12 weeks et al. (2017) women

with PCOS. 30 subjects were given the low-calorie DASH

diet and the other 30

subjects were given a

control diet for 12

weeks. The DASH

and control diets were

consisted of $52-55 \%$

carbohydrates, $30 \%$

total fats, and $16-18 \%$

proteins. Both diets

had equal calorie count. resulted in significant reduction of serum insulin levels $(\mathrm{p}=0.003)$, HOMA-IR score $(p=0.01)$, and serum hs- CRP levels $(p=0.009)$. Additionally, a significant reduction in waist and hip circumference was also seen in the DASH group $(\mathrm{p}=0.003$ and $\mathrm{P}<0.0001$ ), respectively.

12 weeks

Consumption of the DASH diet had a significant reduction in weight $(\mathrm{P}=$ 0.032), BMI $(\mathrm{P}=0.02)$, fat mass $(P=0.008)$, and serum androstenedione ( $\mathrm{P}$ - value $=$ 0.019). An increased in concentrations of SHBG ( $\mathrm{P}=$ 0.003).

DASH diet compared to the control diet had significant decrease in BMI $(p=.02)$, anti-mullerian hormone $(\mathrm{AMH}) \quad(\mathrm{P}=.01), \quad$ insulin $(\mathrm{P}=.02)$, insulin resistance $(\mathrm{P}=.02)$, and free androgen index (FAI) $(\mathrm{P}=.02)$. Significant increase of insulin sensitivity check index $(\mathrm{P}=.02)$, and SHBG $(\mathrm{P}=.01)$. 


\subsection{Results}

The effect of the DASH diet intervention shows potential improvement in BMI and body weight in patients with PCOS. In two studies, BMI and body weight as an outcome variable noted a statistically significant reduction $(p<0.5)$ shown in the DASH diet group compared to the control group (Azadi et al., 2016; Foroozanfard et al., 2017). This is important to point out because a weight loss of more than 5\% can improve symptoms of PCOS in overweight and obese women (Jiskoot et al., 2017). The trials by Asemi et al (2014), Asemi et al (2014), and Foroozanfard et al (2017) had a study length of 8 weeks to 12 weeks and all studies had statistically significant reduction of insulin levels $(p<0.5)$. This is important to note because PCOS is frequently associated with insulin resistance and type II diabetes (Zeng, Xie, Yiu, Long, \& Mo., 2020) and the DASH diet is known to improve type 2 diabetes (cause of insulin resistance) results in hyperinsulinemia (Campbell, 2017).

Homeostatic Model Assessment for Insulin Resistance (HOMA-IR), HOMA-B (B-cell function), and Quantitative Insulin Sensitivity Check Index (QUICKI) are also important highlight because these fasting values show correlation of insulin resistance in patients with PCOS. In Foroozanfard et al (2017) study, results showed a significant reduction $(p<0.5)$ in HOMA-B and non-significant increase in QUICKI; however, in Asemi et al (2014) study it was quite the opposite. No significant reduction mentioned in either QUICKI or HOMA-B when adherence to the DASH eating pattern; however, a significant reduction of HOMA-IR score was seen in the DASH eating pattern compared to the control diet $(p=0.01)$.

Sex hormone binding globin (SHBG) is a transport carrier that binds estrogen and androgens by controlling their biological activities. SHBG was an indicator of hyperandrogenism in women with PCOS (Zhu et al., 2019). SHBF was reduced in obese women with POCS leading to increase in free testosterone levels (Azadi et al., 2016). Low serum of SHBG can indicate abnormal metabolism and are related to insulin resistance, compensatory hyperinsulinemia and irregularities glucose and lipid metabolism in patients with PCOS (Zhu et al., 2019). In Azadi et al (2016) and Foroozandard et al (2017) study, the increased concentration of SHBG had a statistically significant value $(p<0.5)$. Compare to the control diet, the DASH eating pattern resulted in a reduction in serum testosterone and free androgen index $(p<0.5)$; however, after adjustment for potential cofounding variables the difference did not reach a statistically significance (Azadi et al., 2016). Similar to Azadi et al (2016), Foroozandard et al (2017) show significant beneficial effect on anti-mullerian hormone, markers of insulin metabolism, BMI, and FAI; however, did not observe any significant effect following the DASH diet on metabolic profiles (testosterone, HOMA-B, and QUICKI). Likewise, Asemi et al (2014) study had beneficial effects on insulin and triglycerides but no significant difference between the control and DASH diet group when comparing serum total cholesterol, HDL-C and LDL-C.

Overall, the research showed positive results in terms of short terms study (8-12 weeks) in favor of DASH diet for patients with PCOS. The DASH diet, when compared to the control diet, showed improvement in BMI, body weight, HOMA-IR, and SHBG in a study with a short period of time. As mentioned in Jiskoot et al. (2017) study, at least 5\% of weight loss can improve the symptoms of polycystic ovary syndrome. 


\section{Hypocaloric/ Restricted Energy}

Calorie restriction is an established treatment for reducing obesity and lowering type 2 diabetes (Francois et al. (2018). A recent long-term study determined a combination of caloric restriction and exercise to stimulate weight loss of $6 \%-8 \%$ can demonstrate double the improvement in insulin sensitivity compared to the same weight loss by exercise or diet alone (Francois et al. (2018). The development of type 2 diabetes was mainly characterized by insulin resistance that contributes to hyperinsulinemia; therefore, caloric restriction and adding exercise may be coactive as both independently reduce insulin resistance (Francois et al. (2018).

Researcher Van Dam et al (2004) noted the caloric restriction and weight loss often improve excessive androgen production and restore ovulatory cyclicity in 50\%-80\% of obese PCOS patients. This proves Jiskoot et al (2017) outcome of 5\%-10\% of weight loss showed improvement of PCOS symptoms in overweight and obese patients. The main outcome to relief symptoms of PCOS presume to be weight loss followed by improvement of insulin sensitivity; however, Moran et al (2019) study suggested PCOS causes hyperinsulinemia and the inability of insulin to function normally is the outcome of weight gains. Therefore, it is important to consider weight loss as the first line of treatment for PCOS; nonetheless, dietary intervention such as energy restriction can help reduce circulating insulin levels and hyperandrogenism (Moran et al., 2019).

By definition, a low-calorie diet, hypocaloric, or restricted energy is defined as 5000-6000 kJ or around 1200-1400 calories per day (Esfahanian et al., 2012; Moran et al., 2019; Ornstein et al., 2011; Thompson et al., 2008; Thompson et al., 2012; Van Dam et al., 2004). Conversely, Moini et al. (2018) study utilized a recommended total caloric intake be reduced by at least $1000 \mathrm{kcal} / \mathrm{d}$ over 12 weeks period. Three of the studies compared aerobic exercise to hypocaloric diet in achieving results (Moran et al., 2019; Thompson et al., 2008; Thompson et al., 2012), while Ornstein et al. (2011) had two diet treatment groups of low calories versus low fat and Van Dam et al. (2004) tested weight loss based on low calorie diet. Esfahanian et al (2012) was more concerned over the efficacy of Metformin (treat type 2 diabetes) compared with a hypocaloric diet. Recommending 1000 calories or up to 1400 calories per day does not seem like a long-term goal. There is limited research to support hypocaloric diet to treat PCOS long term; therefore, due to different variable outcomes reported more research is needed. Likewise, low calorie diet is best for weight loss in PCOS due to lower energy intake but does not help with insulin resistance or any clinical or biochemical factors (Swora-Cwynar et al., 2016).

\subsection{Clinical Trials}

The description of hypocaloric/ restricted energy diet intervention in studies with participants with PCOS had a variety of study length shown in Table 2. Most studies averaged around 12 weeks (Esfahanian et al., 2012; Moini et al., 2018; Ornstein et al., 2011) while some studies were 20 weeks (Thompson et al., 2008; Thompson et al., 2012), the shortest study duration was 8 weeks (Van Dam et al., 2004), and the longest study duration were 32 weeks (Moran et al., 2019). In Moran et al (2019) study, presented different results at different time frame. At 8 weeks, there 


\section{Ml Macrothink}

were significant change in weight from baseline to 2 months $(p<0.001)$ or over the entire intervention of 32 weeks (Moran et al., 2019). Of two hundred and twenty-one overweight/obese women, one hundred and four women dropped out of the weight loss interventions at different times from 2-8 weeks of 32-week intervention. Likewise, Ornstein et al (2011) had sixteen participants complete the study of twenty-four participants. The reason for the high drop-out includes not enough time for the visits or no longer desire to be in a diet (Ornstein et al., 2011). Nonetheless, multiple studies had majority of their participants completed the intervention (Esfahanian et al., 2012; Moini et al., 2018; Thompson et al., 2008; Thompson et al., 2012).

Other study designs begin to compare diet intervention only or diet intervention with aerobic exercise (Moran et al., 2019; Thompson et al., 2008; Thompson et al., 2012; Van Dam et al., 2004) which led to significant outcomes. The diet provided in multiple studies had a macronutrient breakdown of $30 \%$ of protein, $40 \%$ of carbohydrate, $30 \%$ of fat intake with $6000 \mathrm{~kJ} /$ day (Thompson et al., 2008; Thompson et al., 2012) and were the only two studies that had aerobic exercise with intensity that progressed from 25-30 minutes- 45 minutes. Apart of the energy-restricted diet, the intervention with exercise had a walking/jogging program for five sessions per week. (Thompson et al., 2008; Thompson et al., 2012).

Van Dam et al. (2004) was the only study focused on occurrence of ovulation. Despite the participants achieving a 10\% weight loss about 6 women did not show any signs of ovulation after

participating in a low-calorie diet. Van Dam et al (2004) study consists of a hypocaloric liquid diet- Modifast and Nutridrink with a macronutrient composition of $43 \%$ protein, $15 \%$ fat, $42 \%$ carbohydrate. The study reveals obese women with PCOS resume ovulation in response to caloric restriction; however, weight loss did not differ at baseline except for higher mean SHBG concentrations.

Esfahanian et al. (2012) was the only study that showed statically significant $(p=0.008)$ value results due to incorporating metformin. Comparing both treatment group both had statically significant BMI, waist circumference, fasting insulin, free testosterone, CRP, HOMA, and QUICKI. All biomarkers are important to detect PCOS (Esfahanian et al., 2012). The study results could improve by combining hypocaloric diet with Metformin to enhance the reduction of obesity and insulin resistance. Overall, the study design attempted to demonstrate more significant outcome; however, the biggest outcome for the majority of hypocaloric/ low calorie finding were weight loss through 1200-1400 calories per day. Although, incorporating lifestyle changes like aerobic exercise had better statistically significant outcomes. Table 2 shows the nutrition intervention in studies with participants with PCOS. Table 2 notes the number of participants in each study, the study duration, and the types of outcome expected, and include any aerobic exercise or drug intervention along with the diet intervention. 


\section{Macrothink}

Table 2. Description of hypocaloric/ restricted energy diet interventions in studies with participants with polycystic ovary syndrome

Description of hypocaloric diet interventions in studies of participants with Polycystic Ovary Syndrome

\section{Reference Population}

Esfahanian

40 women with a BMI of $>$ et al. (2012) 27 with PCOS. Receive either Metformin or hypocaloric diet. The patients were assessed before and after treatment for high sensitivity CRP and markers of insulin resistance, fasting glucose to insulin ratio, HOMA, QUICKI.

Moini et al. 90 overweight and obese (2018) women with PCOS had reproductive impairment. $\mathrm{BMI}>28$, age criteria $18-40$

\section{Study Length}

12 weeks

e


clinical trial for weight loss.

The intervention consists of energy restricted diet and/or exercise for 2-8 months.
Ornstein et 24 females age 12-22 years al. (2011) old with PCOS and a BMI above $85^{\text {th }}$ percentile. Compare the effects of a hypocaloric low-fat diet with a very low carbohydrate diet.
12 weeks 2021, Vol. 10, No. 1

attrition was $47.1 \%$ and more than $5 \%$ of weight loss occurred in $63.5 \%$ at 2 months and $62.7 \%$ of weight loss at the end of the study. Despite high attrition due to attendance, weight loss was achieved by $63 \%$ of women with polycystic ovary syndrome in this clinical research setting. Concluded that higher attendance of this study would result in lower attrition and greater weight loss success.

16 participants completed the study, the number of periods increased over 3 months had a statically significant value $\mathrm{P}=0.003$. Weight loss average and waist circumference were also decreased $(\mathrm{p}<0.0001$ and $\mathrm{p}=0.01$, respectively). Those with weight loss were 3.4 times more likely to have improved menstrual function $(p=0.001)$. NO statistically significant differences between the two groups.

Thompson The study enrolled 9420 weeks et al. (2008) overweight and obese women with PCOS with a mean age of 29.3 and a body mass index of 36.1. 30 subjects were randomized to a diet only, 31 subjects to a diet and aerobic exercise, and 33 subjects were given
All treatment was determined to reduce weight loss with no difference between each group. Fat-free mass and fat mass decreased more in those enrolled in the diet and aerobic exercise and diet and combined aerobic-resistance exercise. Other biochemical 
a diet and combined aerobic-resistance exercise.
Thompson $50 \quad$ overweight/obese

et al. (2012) women with PCOS from a previous study. The subjects enrolled are randomized by a computer generator to one of the 3 treatments. 14 subjects were advised to consume $6000 \mathrm{~kJ} /$ day, 16 subjects were advised to go on the diet with aerobic exercise and the remaining 16 subjects had a diet and combined aerobic-resistance exercise. Weight, endothelial function, intercellular adhesion, plasminogen activator inhibitor, insulin resistance, and hormonal profile were assessed at baseline and at the end of the intervention.

Van Dam et 15 hyperandrogenemia al (2004) oligoovulatory patients that underwent a low-calorie diet where they showed $10 \%$ weight loss or more within 6.25 months.

values had no difference between treatments but showed improvement (free androgen index $\mathrm{p}<0.001)$ The study concluded that with restricted diet and aerobic exercise can improve body composition but no further effect in cardiometabolic, hormonal, and reproduction function was related to diet alone.

20 weeks

All three treatments had significant weight loss of a $\mathrm{P}$ value greater than 0.001 . Insulin resistance, testosterone, sex hormone-binding globulin and the free androgen index showed improvement but no differences between the treatment. The study concluded that exercise training did not prove to be an additional benefit to following the hypocaloric diet/ high protein over the markers of endothelial function when it comes to overweight/obese women with PCOS.

\section{8 weeks}

All women except one lost $10 \%$ body weight. BMI of the responder's group had decrease but non statistically significant value. Other biochemicals factors show 
Hormones were measured at baseline, after one week of the low-calorie diet and after $10 \%$ weight loss. significant decline was free testosterone index, HOMA-IR, and insulin and a rise in SHBG in response to weight loss.

\subsection{Results}

Weight loss was the only variable which had statistically significant value $(p<0.001)$ at two months; however, body mass index, waist circumference, fasting glucose, homeostatic model assessment for insulin resistance, testosterone, sex hormone binding globin, free androgen index, and menstrual irregularity had no statistically significant value at 2 months or at the end of study duration (Moran et al., 2019). Moran et al (2019) interventions included an energy restricted or an energy restricted with aerobic exercise; however, results did not explain the outcome of which intervention had better improvement of PCOS. The study main outcome was significant in the record of weight loss were shown at 2 months (Van Dam et al., 2004; Moran et al., 2019). There were no statistically significant differences between the diet only group or diet with exercise group. All treatment resulted in weight loss $(p<0.001)$ across all studies (Esfahanian et al., 2012; Moini et al., 2018; Ornstein et al., 2011; Moran et al., 2019; Thompson et al., 2008; Thompson et al., 2012; Van Dam et al., 2004).

Moran et al (2019) was the only study that did not find statistically significant results for waist circumference; however, successful weight loss was achieved by $63 \%$ of women with PCOS. Most studies with diet intervention plus aerobic exercise or plus metformin had statistically significant levels $(p=0.001)$ of waist circumference, fasting insulin, and free testosterone (Esfahanian et al., 2012; Moini et al., 2018; Ornstein et al., 2011; Thompson et al., 2008; Van Dam et al., 2004). Thompson et al (2012) and Thompson et al (2008) had improvement of free testosterone, glucose, sex hormone binding globin, free androgen index, and insulin resistance $(p=<0.001)$.

Most notably, the study by Moini et al (2019), 72\% responded to the intervention with improvement in menstrual cyclicity while $28 \%$ lack improvement in menstrual regularity. This study had a total daily caloric intake of $1000 \mathrm{kcal} /$ day and the intervention duration was for 12 weeks which had significant $(p<0.001)$ reduction in weight, waist circumference, fasting insulin, and free testosterone. Moini et al (2019) also reported the group with improved menstrual cyclicity had a significant reduction $(p=0.001)$ in AMH level. Improvement of anti-mullerian hormone shows progress of symptoms for patients with PCOS.

\section{Low Glycemic/ Mediterranean Diet/ Low Carbohydrate}

According to the Recommendations from the International Evidence-based Guideline for the Assessment and Management of PCOS, weight management is one of the main treatment strategies (Teede et al., 2018). Nonetheless, the low glycemic/Mediterranean/low carbohydrate diet is still up for discussion on the best effective nutritional pattern people should followed to lose weight for PCOS. Among the different nutritional strategies, the Mediterranean diet is known to promote a healthy eating pattern including: Regular 
consumption of unsaturated fats, low glycemic index carbohydrate, vitamins, fibers, and moderate amount of animal proteins (Barrea et al., 2019). The Mediterranean diet has been reported to have a well-established anti-inflammatory, which derived from high intake of polyunsaturated fatty acids (PUFA) omega 2, fiber, and antioxidants from fruit and vegetables (Barrea et al., 2019). Conversely, low-grade chronic inflammatory status often coexists with polycystic ovary syndrome.

Glycemic index is one of the several values used to measure how rapidly the body breaks down carbohydrate. The Mediterranean diet similar to the low glycemic diet mainly focus on including strong vegetable content and limit carbohydrate, which is susceptible spike of blood glucose. Likewise, it is important to control blood sugar and improve insulin resistance and glucose metabolism. Majority of women with PCOS showed marked compensatory hyperinsulinemia after carbohydrate intake; this highlights the potential advantage to the low glycemic diet due to its limit on carbohydrate ingestion (Marsh et al., 2010). In terms of effective methods to reduce risk factors for PCOS, Marsh et al (2010) predicts weight loss with a low glycemic diet would improve underlying insulin resistance. Unlike Marsh et al (2010), Barr et al (2013) primary outcome was to see changes to the insulin markers and secondary outcomes include body composition and changes in lipids. The goals for Mediterranean diet/ low glycemic diet focus on improving insulin resistance and glucose features (control blood sugar).

Similar to the low glycemic diet and Mediterranean Diet, a diet low in carbohydrate ingestion resulted in reduction of the circulating insulin levels, which promoted high level of fatty acids in the body used for oxidation and the production of ketones bodies (Adam-Perrot et al., 2006). The cycle of low carbohydrate caused metabolic changes in the body where ketones bodies are produced, and the body goes into ketosis (Adam-Perrot et al., 2006). Most common name for this diet is called ketogenic diet; however, due to lack of current clinical trials of the ketogenic diet for patients with PCOS, the combination of low glycemic, Mediterranean Diet, and low carbohydrate are coalesced in this systematic review. The conglomerate of diets mainly focuses on low carbohydrate which facilitate favorable effects such as rapid weight loss, decrease of fasting glucose and insulin levels (Barr et al., 2013; Barrea et al., 2019; Marsh et al., 2010; Mehrabani et al., 2012; Shishehgar et al., 2019; Turner-McGrievy et al., 2014; Gower et al., 2013).

\subsection{Clinical Trials}

Women with PCOS are essentially insulin resistant and are at risk for type 2 diabetes and cardiovascular disease. Weight loss improves these risk factors, but the optimal diet is well unknown. The low glycemic diet is recommended without any clinical effectiveness; therefore, researcher Marsh et al (2010) studied participant outcomes after a low glycemia diet or macronutrient matched a healthy diet for 12 months or until they achieve weight loss of $7 \%$. Weight loss of more than $5 \%$ has an effective improvement in many features of PCOS (Teede et al., 2013). Important inclusion and exclusion criteria included the following: Participants were excluded if patients have type 2 diabetes, current breastfeeding, (Barr et al., 2013; Marsh et al., 2010), age $<18$ years and $>40$ years, occasional use of drugs (metformin or any anti-inflammatory drugs), eating disorder or specific nutrition regimens (Barr et al., 2013; Barrea et al., 2019). Participants were included if patients were diagnosed with PCOS, 
within age range (18-40), not within healthy weight range, and no known intentional weight loss (Marsh et al., 2010).

The length of the clinical trials is notable. The trials vary from 8 weeks to 48 weeks. An 8 -week trial showed statistically significant results $(p<0.05)$, which gives the similar results as a 48-week trial or a 36-week trial (Barr et al., 2013; Gower et al., 2013; Marsh et al., 2010). The difference was the outcomes variable being measured such as body mass index, fasting insulin, testosterone, sex hormone binding globin, free androgen index, waist circumference and HoMA-IR in the intervention. Individual consultation with a Registered Dietitian were held to explain how the diet will be implement and provided diet education for the intervention (Barr et al., 2013; Shishehgar et al., 2019; Turner-McGrievy et al., 2014). To enhance compliance the Registered Dietitian monitored the prescribed diet during regular consultations (Mehrabani et al., 2011; Marsh et al., 2010).

Gower and colleagues' (2013) research had a standard diet of 55\% carbohydrate, $18 \%$ protein, $27 \%$ fat, whereas the treatment group (lower carbohydrate) had a macronutrients composition of $41 \%$ carbohydrate, $19 \%$ protein, and $40 \%$ total fat. In a similar study, Mehrabani et al. (2010) macronutrient of 40\% energy, 30\% protein, and 30\% fat had improved weight loss when compared to $55 \%$ of energy of carbohydrate. However, no significant differences between the two diets. Most notable information included in the intervention is the glycemic index value of the standard diet was approximately 60, whereas the lower carbohydrate was approximately 50 (Gower et al., 2013). Researchers ensured participants were able to access information about foods with a high glycemic index and encouraged to avoid consumption of any high GI foods from the provided booklet (Shishehgar et al., 2019).

The targeted macronutrient in Shishehgar et al. (2019) was 50\% energy from carbohydrate with low to medium glycemic index value, $20 \%$ of protein and $30 \%$ from fat. Participants were instructed to eat lean meat, whole grains, low fat dairy, non-starchy vegetables and were prohibited from eating fast foods or food high in salt (Shishehgar et al., 2019). Tuner-McGrievy et al (2014) examined the effectiveness of two different dietary approaches for weight loss: Vegan diet with low glycemic with no caloric restriction versus a standard low-calorie diet of 1200-1500 kcal/day. The present study found the low glycemic vegan diet had significantly ( $p$ value?) greater weight loss at 3 months and greater dietary intake at six months compared to the low-calorie diet approach (Tuner-McGrievy et al (2014). Table 3 shows the nutrition intervention in studies with participants with PCOS. Table 3 notes the number of participants in each study, the study duration, and the types of outcome expected, and include any aerobic exercise or drug intervention along with the diet intervention. 


\section{Macrothink}

Table 3. Description of Low Glycemic diet/ Mediterranean diet interventions in studies with participants with polycystic ovary syndrome

Description of low glycemic/ Mediterranean diet interventions in studies of participants with Polycystic Ovary Syndrome

\begin{tabular}{llllr}
\hline Reference & Population & $\begin{array}{l}\text { Study } \\
\text { Length }\end{array}$ & $\begin{array}{l}\text { Authors description } \\
\text { nutrition intervention }\end{array}$ & of
\end{tabular}

Barr et al. (2013) 26 women with PCOS 36 recruited for a low GI weeks dietary intervention phase (weeks 0 to 12), (weeks 12 to 24), and (weeks 24 to 36). Participants were asked to ensure their evening meals contained between 30-50 grams of carbohydrate and remained fasted from 8:00pm before every research center visit.

Barrea et al. 224 subjects enrolled to the (2019)

Marsh et al. 96 (2010) overweight/obese 48 study. There are two treatment group:112 women with PCOS and 112 without PCOS. Seven-day food records were used to evaluate the degree of adherence to the MD dietary pattern.

4 years

PCOS women showed higher testosterone levels, fasting insulin, glucose levels, HoMA-IR compared to women without PCOS $(p<0.001)$. PCOS women had a lower adherence to the MD diet than the control group $(p<0.001)$. Women with PCOS with an average adherence to the MD diet showed statistically significant value $(p=0.004)$.

premenopausal women weeks with PCOS to consume either an ad libitum low-GI diet or a macronutrient matched healthy diet until they
The attrition rate was high in both treatment groups. Among the groups that completed the study the low GI diet group had more improvement than the conventional health diet $(p=$ 0.03 ). With metformin and low 
achieved a 7\% weight loss

Mehrabani et al. 60 overweight and obese (2012)

Shishehgar et al (2019)

Turner-McGrievy et al. (2014) women with PCOS who insulin-sensitizing agents were assigned to different treatment group: hypocaloric diet $(15 \%$ protein) or a modified hypocaloric diet with high protein and low glycemic load (30\% protein).

62 participants (28 with PCOS and 34 patients without PCOS) followed a 24-week energy restricted LGI diet. did not use
12 weeks 2021, Vol. 10, No. 1

GI diet, there were greater improvement $(p=0.048)$ and had better menstrual cyclicity $(p$ $=0.03$ )

In both groups led a significantly reduced body weight and androgen levels; however, the combination of high protein and low glycemic load foods had a better increase in insulin sensitivity and decrease in hs-CRP levels.

24

weeks

Both groups (with and without PCOS) achieved weight loss; however, did not differ significantly. No significance difference between HOMA-IR and insulin resistance; however, reduction in total testosterone ( $p$ $<0.001)$ and increase in SHBG $(\mathrm{p}<0.001)$ were observed. Menstrual irregularity was improved by $80 \%$ of women with PCOS and there was a significant decrease in occurrence of acne in the group with PCOS after the energy restricted diet.

The result of the study concluded that attrition was high at 3 months of $39 \%$ while at 6 months they were a $67 \%$ improvement. The vegan group with low glycemic demonstrated more weight loss at 6 months than the low-calorie, but there 
glycemic would be more effective than the low-calorie diet. was no significant difference between the two groups at 6 months. Concluded that low glycemic vegan may be effective for short term weight loss in women with PCOS.

Gower et al. 30 women with PCOS 8 weeks Low $\mathrm{CHO}$ had significant (2013) enrolled into two different eucaloric diets differing in CHO composition (41\% vs $55 \%$ )

decreases in B-cell response, fasting insulin, fasting glucose, HoMA-IR, total testosterone and significant increase in insulin sensitivity. Across all data, the change in testosterone was positively associated with the changes in fasting insulin $(\mathrm{P}<$ 0.05 )

\subsection{Results}

Barr et al (2013) investigated the low glycemic index diet and the outcomes during the study duration of 0-12 weeks, 12-24 weeks, and 24-36 weeks. While significant decreases $(p>0.05)$ in dietary glycemic index was achieved in a 12-week intervention no difference was found to metabolic risk makers and body composition. At 36 weeks, body composition remains unsignificant; however, insulin sensitivity did improve after intervention $(p=0.03)$. Marsh et al (2010), demonstrate higher weight loss $(p=0.03)$ and increase in menstrual regularity $(p=$ $0.03)$ in the low glycemic index group compared to the conventional healthy group treatment. Insulin sensitivity increased significantly $(p<0.01)$ in the low-GI diet group (Marsh etal., 2010; Bar et al., 2013). Adherence to the low glycemic index diet presents significantly $(p<$ 0.001) higher CRP, testosterone levels, HoMA-IR than participants with moderate to high-GI intake (Barrea et al., 2019).

The most notable information in the low glycemic index/low carbohydrate diet is the outcome of anthropometrics such as waist circumference, and hip circumference. Body composition remains non statistically significant with the low glycemic index diet. There is significant weight loss between the two-treatment groups $(p<0.05)$; however, one treatment group with the low-carbohydrate tends to have higher weight loss at the end of the study duration (Mehrabani et al., 2010; Shishehgar et el., 2019; Turner-McGrievy et al., 2014; Gower et al., 2013). This could potentially explain a study can have better effect with longer study duration.

In Gower et al. (2014) study, the consumption of a lower carbohydrate diet resulted in significant decreases in HoMA-IR, fasting insulin $(p<0.001)$, fasting glucose $(p<0.01)$, testosterone $(p<\mathrm{XXX})$, and increases in insulin sensitivity $(p<0.05)$ in the 8 weeks duration. The fundamental goals of the low carbohydrate diet intervention were to improve fasting 
insulin $(p<0.001)$ and lowering testosterone $(p<0.05)$ by way of lowering insulin; however, SHBG did not approach significance. Thus, the data collected suggested manipulation of insulin can have positive effects in testosterone in women with PCOS (Gower et al., 2014). Polycystic Ovary Syndrome is the cause of ovulatory infertility, which produce high levels of circulating androgens such as testosterone and this interfere with menstrual cycle. Shishehgar et al. (2019) outcome of menstrual irregularity was improved by $80 \%$ and statistically significant $(p=0.03)$ in Marsh et al. (2010).

Overall, low glycemic index /low carbohydrate (less than $41 \%$ carbohydrate intake) was associated with significant improvement in measures of carbohydrate metabolism results in better insulin biomarkers. Compared to the different treatment groups, low glycemic index diet highlighted improvements in insulin sensitivity, fasting insulin, fasting glucose, and testosterone than the clinical anthropometric features such as waist circumference, hip circumference (Mehrabani et al., 2010; Shishehgar et el., 2019; Turner-McGrievy et al., 2014; Gower et al., 2013; Marsh et al., 2010; Bar et al., 2013; Barr et al., 2013).

\section{Discussion}

Many studies have addressed weight loss and improvement of insulin resistance/insulin sensitivity in their clinical trials. The consumption of the DASH eating pattern compared to other control diet resulted in an improvement reduction in body weight, HoMA-IR, improvement in BMI, SHBG, AMH, and insulin metabolism (Asemi et al., 2014); Asemi et al., 2014; Azadi et al., 2016; Foroozanfard et al., 2017). The studies have a sample size of less than 60 PCOS subjects, which is a limitation. Moreover, each study varies considerably in design, intensity, and variable outcome measures; therefore, conclusive results remain elusive. The exact causes of PCOS are still unclear. PCOS is a common endocrine disorder described as a series of hormonal and metabolic issues such as insulin resistance and reduction of insulin sensitivity. PCOS also affects fertility issues caused by ovarian hyperandrogenism (Dennett et al., 2015). Nonetheless, one might prompt that the effect of DASH diet on insulin sensitivity is dependent on the effect of weight loss; however, Asemi and colleagues' (2014), controlled the weight loss and results of insulin sensitivity remains significant. This signify the effect of DASH diet on insulin resistance was independently not affected by body weight (Asemi et al., 2014).

After reviewing the hypocaloric diet, calorie restriction is well known as a treatment for reducing obesity and lowering type II diabetes. PCOS is an independent risk factor for impaired glucose tolerance and insulin resistance. Implementation of exercise along with hypocaloric diet intervention may be interdependent as both independently reduce insulin resistance. Francois et al and this team (2018) demonstrated a combination of low-calorie diet and interval exercise (INT) which improves glucose tolerance more than low calorie diet alone. With the caloric restriction of 1200-1500 kcals/day, majority of the studies included in this review had participants dropped out before the conclusion of the study period (Esfahanian et al., 2012; Moran et al., 2015; Van Dam et al., 2004) due to no desire being in the intervention (Ornstein et al., 2011). In Thompson et al (2012) and Thompson et al (2008) the improvement of free testosterone, glucose, sex hormone binding globin, free androgen index, and insulin resistance $(p=<0.001)$ were noted in their study. In Jeanes, Y.M, S. Barr, K. Smith, K.H. Hart. (2009) confirms that while women are aware of their PCOS condition 


\section{Ml Macrothink}

and recognize the importance of diet and lifestyle, their resources are coming from an unreliable source).

While following a low-calorie diet could potentially improve free testosterone and insulin resistance, Thompson et al. (2008), Thompson et al. (2012), and Szczuko et al. (2019) proposed following a low-calorie with a lower glycemic can silence inflammation in patients with PCOS. In recent years, further studies explained inflammation processes involved in ovulation played an important role in ovarian follicular dynamics (Rostamtabar et al., 2020). In the low carbohydrate/ low glycemic diet the most notable findings are fasting insulin and testosterone levels. Most studies had a reduction in body weight, which further automatically lead to an improvement in some of the symptoms and risk factors of PCOS. Elsheikh (2013) mentioned compared to the low glycemic diet, the low carbohydrate diet achieved greater degree of improvement in weight, body composition, lipid, insulin and testosterone levels however did not reach statistical significance value. Nonetheless, following a low glycemic index diet showed improvement in insulin sensitivity, fasting insulin, fasting glucose, and testosterone than the clinical anthropometric features such as waist circumference, hip circumference (Mehrabani et al., 2010; Shishehgar et el., 2019; Turner-McGrievy et al., 2014; Gower et al., 2013; Marsh et al., 2010; Bar et al., 2013; Barr et al., 2013).

Consumption of a diet high in fruits, vegetables, whole grains, and legumes reduced oxidative stress and inflammation (Johnston., 2019), which almost replicated the Mediterranean diet. Other important factors for improvement for PCOS is to make sure to reduce stress levels and maintain adequate amounts of sleep which can decrease hirsutism and total testosterone (Jamillian et al, 2019). An important element to note is the low 25 (OH) D levels may influence both insulin secretion and insulin sensitivity in PCOS; therefore, adequate supplementation of vitamin D is crucial for women with PCOS (Lin \& Wu, 2015).

\subsection{Limitations of the Research}

A notable limitation to this systematic review is the lack of recent clinical trials. Most clinical trials were published longer than 5 years ago. There are no recent studies, to know of the writer, addressing to compare DASH diet versus hypocaloric diet or Mediterranean versus DASH diet, while assessing long term results. Additionally, each study varied considerably in design, intensity, and variable outcome measures; therefore, results remain inconclusive. Each clinical trial had different control and treatment groups and different variable outcomes measures. More research is warranted to compare the DASH diet versus hypocaloric or hypocaloric versus low glycemic index or low carbohydrate and assess the long-term results.

\section{Conclusions}

The literature review followed different research studies exploring the DASH, Mediterranean diet/low glycemic index/low carbohydrate, and hypocaloric diet in finding possible outcomes to relief symptoms of polycystic ovary syndrome. Not only is the DASH diet a great intervention for hypertension, but the DASH diet also benefits overweight and obese women in the improvement of weight loss in polycystic ovary syndrome. This literature review supports the DASH diet to improve blood lipid profiles, glycemic index, sex hormones, and insulin sensitivity (Azadi-Yazdi et al., 2016; Asemi et al., 2014; Foroozanfard et al., 2017). Presently, the Mediterranean/low glycemic index/ low carbohydrate diet established an 


\section{MInstitute ${ }^{\text {Macrothink }}$}

improved in health outcomes such as decreased inflammation and prevalence of metabolic syndrome, depression, abnormal glucose tolerance, and regulated menstrual irregularity among women with PCOS (Shishehgar et al., 2019; Barrea et al., 2019; Moran et al., 2015). The hypocaloric/ energy restricted diet promotes weight loss and in the majority of the studies improved menstrual function. While an energy restricted diet plus exercise is ideal for women with PCOS, the energy restricted diet improves the regulation of the menstrual cycle as well as insulin sensitivity (Thompson et al., 2008; Thompson et al., 2012; Esfahanian et al., 2012; Stamets et al., 2004). One of the major research findings by following a low-calorie diet was the improvement in anthropometric parameters such as: Body weight, BMI, and waist circumference (Esfahanian et al., 2012; Moini et al., 2018; Ornstein et al., 2011; Moran et al., 2019; Thompson et al., 2008; Thompson et al., 2012; Van Dam et al., 2004). Body fat content was reduced while significant increases in lean body percentage were noted (Esfahanian et al., 2012; Moini et al., 2018; Ornstein et al., 2011; Moran et al., 2019; Thompson et al., 2008; Thompson et al., 2012; Van Dam et al., 2004). This systematic review provided a proof of a consistent weight loss in all three different diet intervention for overweight/obese women with polycystic ovary syndrome; however, each study varies significantly in design and variable outcome measures. A DASH diet and a low glycemic index/Mediterranean diet may be the most effective starting point to achieve weight loss in PCOS, and then consider long term interventions of weight loss or improvement in insulin resistance through more research in the future.

\section{Acknowledgement}

The author would like the thank Dr. Haubrick for his support and guidance. She would also like to thank her family for their everlasting support, encouragement, and love.

\section{References}

Adam-Perrot, A., Clifton, P., \& Brouns, F. (2006). Low-carbohydrate diets: nutritional and physiological aspects. Obesity Reviews, 7(1), 49-58. https://doi.org/10.1111/j.1467-789x.2006. 00222.x

Asemi, Z., \& Esmaillzadeh, A. (2014). DASH Diet, Insulin Resistance, and Serum hs-CRP in Polycystic Ovary Syndrome: A Randomized Controlled Clinical Trial. Hormone and Metabolic Research, 47(03), 232-238. https://doi.org/10.1055/s-0034-1376990

Asemi, Z., Samimi, M., Tabassi, Z., Shakeri, H., Sabihi, S.-S., \& Esmaillzadeh, A. (2014). Effects of DASH diet on lipid profiles and biomarkers of oxidative stress in overweight and obese women with polycystic ovary syndrome: A randomized clinical trial. Nutrition, 30(11-12), 1287-1293. https://doi.org/10.1016/j.nut.2014.03.008

Azadi-Yazdi, M., Karimi-Zarchi, M., Salehi-Abargouei, A., Fallahzadeh, H., \& Nadjarzadeh, A. (2016). Effects of Dietary Approach to Stop Hypertension diet on androgens, antioxidant status and body composition in overweight and obese women with polycystic ovary syndrome: a randomised controlled trial. Journal of Human Nutrition and Dietetics, 30(3), 275-283. https://doi.org/10.1111/jhn.12433

Barr, S., Reeves, S., Sharp, K., \& Jeanes, Y. M. (2013). An Isocaloric Low Glycemic Index Diet Improves Insulin Sensitivity in Women with Polycystic Ovary Syndrome. Journal of the Academy of Nutrition and Dietetics, 113(11), 1523-1531. https://doi.org/10.1016/j. 
jand.2013.06.347

Barrea, L., Arnone, A., Annunziata, G., Muscogiuri, G., Laudisio, D., Salzano, C., ... Savastano, S. (2019). Adherence to the Mediterranean Diet, Dietary Patterns and Body Composition in Women with Polycystic Ovary Syndrome (PCOS). Nutrients, 11(10), 2278. https://doi.org/10.3390/nu11102278

Campbell, A. P. (2017). DASH Eating Plan: An Eating Pattern for Diabetes Management. Diabetes Spectrum, 30(2), 76-81. https://doi.org/10.2337/ds16-0084

Costello, M. F., Shrestha, B., Eden, J., Johnson, N., \& Moran, L. J. (2007). Insulin-sensitising drugs versus the combined oral contraceptive pill for hirsutism, acne and risk of diabetes, cardiovascular disease, and endometrial cancer in polycystic ovary syndrome. Cochrane Database of Systematic Reviews. https://doi.org/10.1002/14651858.cd005552.pub2

Costello, M. F., Misso, M. L., Wong, J., Hart, R., Rombauts, L., Melder, A., ... Teede, H. J. (2012). The treatment of infertility in polycystic ovary syndrome: a brief update. Australian and New Zealand Journal of Obstetrics and Gynaecology, 52(4), 400-403. https://doi.org/10. 1111/j.1479-828x.2012.01448.x

Dennett, C. C., \& Simon, J. (2015). The role of polycystic ovary syndrome in reproductive and metabolic health: overview and approaches for treatment. Diabetes spectrum: $a$ publication of the American Diabetes Association, 28(2), 116-120. https://doi.org/10.2337/ diaspect.28.2.116

Elsheikh, M. (2013). Randomised trial comparing a Low Carbohydrate Diet and a Low Glycaemic Index Diet on body weight, hyperandrogenism and cardiovascular risk factors in women with Polycystic Ovary Syndrome (PCOS). https://doi.org/10.1186/isrctn55425758

Escobar-Morreale, H. F. (2018). Polycystic ovary syndrome: definition, aetiology, diagnosis and treatment. Nature Reviews Endocrinology, 14(5), 270-284. https://doi.org/10.1038/nrendo. 2018.24

Esfahanian, F., Zamani, M. M., Heshmat, R., \& Moini nia, F. (2012). Effect of Metformin compared with hypocaloric diet on serum C-reactive protein level and insulin resistance in obese and overweight women with polycystic ovary syndrome. Journal of Obstetrics and Gynaecology Research, 39(4), 806-813. https://doi.org/10.1111/j.1447-0756.2012.02051.x

Francois, M., Gilbertson, N., Eichner, N., Heiston, E., Fabris, C., Breton, M., ... Malin, S. (2018). Combining Short-Term Interval Training with Caloric Restriction Improves B-Cell Function in Obese Adults. Nutrients, 10(6), 717.

Francois, M. E., Gilbertson, N. M., M Eichner, N. Z., Heiston, E. M., Mehaffey, J. H., Hassinger, T. E., ... Malin, S. K. (2018). Combining Short-Term Interval Training With Caloric-Restriction Improves B-Cell Function In Obese Adults. Medicine \& Science in Sports \& Exercise, 50(5S), 625. https://doi.org/10.1249/01.mss.0000537151.60457.85

Foroozanfard, F., Rafiei, H., Samimi, M., Gilasi, H. R., Gorjizadeh, R., Heidar, Z., \& Asemi, Z. (2017). The effects of dietary approaches to stop hypertension diet on weight loss, anti-Müllerian hormone and metabolic profiles in women with polycystic ovary syndrome: A randomized clinical trial. Clinical Endocrinology, 87(1), 51-58. https://doi.org/10.1111/ cen. 13333 


\section{MInstitute Macrothink $_{\text {Int }}$}

Gower, B. A., Chandler-Laney, P. C., Ovalle, F., Goree, L. L., Azziz, R., Desmond, R. A., ... Bates, G. W. (2013). Favourable metabolic effects of a eucaloric lower-carbohydrate diet in women with PCOS. Clinical Endocrinology, 79(4), 550-557. https://doi.org/10.1111/cen. 12175

Jamilian, M., Foroozanfard, F., Mirhosseini, N., Kavossian, E., Aghadavod, E., Bahmani, F., ... Asemi, Z. (2019). Effects of melatonin supplementation on hormonal, inflammatory, genetic, and oxidative stress parameters in women with polycystic ovary syndrome. Frontiers in Endocrinology, 10.

Jeanes, Y. M., Barr, S., Smith, K., \& Hart, K. H. (2009). Dietary management of women with polycystic ovary syndrome in the United Kingdom: the role of dietitians. Journal of Human Nutrition and Dietetics, 22(6), 551-558. https://doi.org/10.1111/j.1365-277x.2009.00988.x

Johnston, C. (2009). Functional Foods as Modifiers of Cardiovascular Disease. American Journal of Lifestyle Medicine, 3(1_suppl). https://doi.org/10.1177/1559827609332320

Love, J., McKenzie, J., Nikokavoura, E. A., Broom, I., Rolland, C., \& Johnston, K. (2016). The experiences of women with polycystic ovary syndrome on a very low-calorie diet. International Journal of Women's Health, Volume 8, 299-310. https://doi.org/10.2147/ijwh. s100385

Marsh, K. A., Steinbeck, K. S., Atkinson, F. S., Petocz, P., \& Brand-Miller, J. C. (2010). Effect of a low glycemic index compared with a conventional healthy diet on polycystic ovary syndrome. The American Journal of Clinical Nutrition, 92(1), 83-92. https://doi.org/10. 3945/ajen.2010.29261

Mathur, R., Alexander, C. J., Yano, J., Trivax, B., \& Azziz, R. (2008). Use of metformin in polycystic ovary syndrome. American Journal of Obstetrics and Gynecology, 199(6), 596-609. https://doi.org/10.1016/j.ajog.2008.09.010

Mayhew, M. S. (2011). Treatment for Polycystic Ovary Syndrome. The Journal for Nurse Practitioners, 7(6), 517-518. https://doi.org/10.1016/j.nurpra.2011.04.009

Mehrabani, H. H., Salehpour, S., Amiri, Z., Farahani, S. J., Meyer, B. J., \& Tahbaz, F. (2012). Beneficial Effects of a High-Protein, Low-Glycemic-Load Hypocaloric Diet in Overweight and Obese Women with Polycystic Ovary Syndrome: A Randomized Controlled Intervention Study. Journal of the American College of Nutrition, 31(2), 117-125. https://doi.org/10.1080/ 07315724.2012.10720017

Moher, D., Shamseer, L., Clarke, M., Ghersi, D., Liberati, A., Petticrew, M., ... Stewart, L. A. (2015). Preferred reporting items for systematic review and meta-analysis protocols (PRISMA-P) 2015 statement. Systematic Reviews, 4(1). https://doi.org/10.1186/ 2046-4053-4-1

Moini, A., Arabipoor, A., Hemat, M., Ahmadi, J., Salman-Yazdi, R., \& Zolfaghari, Z. (2018). The effect of weight loss program on serum anti-Müllerian hormone level in obese and overweight infertile women with polycystic ovary syndrome. Gynecological Endocrinology, 35(2), 119-123. https://doi.org/10.1080/09513590.2018.1499084

Moran, L., Noakes, M., Clifton, P., Buckley, J., Brinkworth, G., Thomson, R., \& Norman, R. (2019). Predictors of Lifestyle Intervention Attrition or Weight Loss Success in Women with Polycystic Ovary Syndrome Who Are Overweight or Obese. Nutrients, 11(3), 492. 


\section{MInstitute ${ }^{\text {Mink }}$}

https://doi.org/10.3390/nu11030492

Ornstein, R. M., Copperman, N. M., \& Jacobson, M. S. (2011). Effect of Weight Loss on Menstrual Function in Adolescents with Polycystic Ovary Syndrome. Journal of Pediatric and Adolescent Gynecology, 24(3), 161-165. https://doi.org/10.1016/j.jpag.2011.01.002

Rasool, S. U., Ashraf, S., Nabi, M., Rashid, F., Fazili, K. M., \& Amin, S. (2019). Elevated fasting insulin is associated with cardiovascular and metabolic risk in women with polycystic ovary syndrome. Diabetes \& Metabolic Syndrome: Clinical Research \& Reviews, 13(3), 2098-2105. https://doi.org/10.1016/j.dsx.2019.05.003

Rostamtabar, M., Esmaeilzadeh, S., Tourani, M., Rahmani, A., Baee, M., Shirafkan, F., ... Nouri, H. R. (2020). Pathophysiological roles of chronic low-grade inflammation mediators in polycystic ovary syndrome. Journal of Cellular Physiology, 236(2), 824-838. https://doi.org/10.1002/jcp.29912

Shishehgar, F., Mirmiran, P., Rahmati, M., Tohidi, M., \& Ramezani Tehrani, F. (2019). Does a restricted energy low glycemic index diet have a different effect on overweight women with or without polycystic ovary syndrome? BMC Endocrine Disorders, 19(1). https://doi.org/10.1186/s12902-019-0420-1

Standards of Medical Care in Diabetes--2012. (2011). Diabetes Care, 35(Supplement_1). https://doi.org/10.2337/dc12-s011

Stamets, K., Taylor, D. S., Kunselman, A., Demers, L. M., Pelkman, C. L., \& Legro, R. S. (2004). A randomized trial of the effects of two types of short-term hypocaloric diets on weight loss in women with polycystic ovary syndrome. Fertility and Sterility, 81(3), 630-637.

Swora-Cwynar, E., Kujawska-Łuczak, M., Suliburska, J., Reguła, J., Kargulewicz, A., Kręgielska-Narożna, M., ... Bogdański, P. (2016). The effects of a low-calorie diet or an isocaloric diet combined with metformin on sex hormones in obese women of child-bearing age. Acta Scientiarum Polonorum Technologia Alimentaria, 15(2), 213-220.

Szczuko, M., Zapalowska-Chwyć, M., \& Drozd, R. (2019). A Low Glycemic Index Decreases Inflammation by Increasing the Concentration of Uric Acid and the Activity of Glutathione Peroxidase (GPx3) in Patients with Polycystic Ovary Syndrome (PCOS). Molecules, 24(8), 1508. https://doi.org/10.3390/molecules24081508

Thomson, R. L., Brinkworth, G. D., Noakes, M., Clifton, P. M., Norman, R. J., \& Buckley, J. D. (2012). The effect of diet and exercise on markers of endothelial function in overweight and obese women with polycystic ovary syndrome. Human Reproduction, 27(7), 2169-2176. https://doi.org/10.1093/humrep/des 138

Thomson, R. L., Buckley, J. D., Noakes, M., Clifton, P. M., Norman, R. J., \& Brinkworth, G. D. (2008). The Effect of a Hypocaloric Diet with and without Exercise Training on Body Composition, Cardiometabolic Risk Profile, and Reproductive Function in Overweight and Obese Women with Polycystic Ovary Syndrome. The Journal of Clinical Endocrinology \& Metabolism, 93(9), 3373-3380. https://doi.org/10.1210/jc.2008-0751

Teede, H. J., Joham, A. E., Paul, E., Moran, L. J., Loxton, D., Jolley, D., \& Lombard, C. (2013). Longitudinal weight gain in women identified with polycystic ovary syndrome: Results of an observational study in young women. Obesity, 21(8), 1526-1532. https://doi.org/10.1002/oby.20213 


\section{Macrothink}

Teede, H. J., Misso, M. L., Costello, M. F., Dokras, A., Laven, J., Moran, L., ... Yildiz, B. O. (2018). Recommendations from the international evidence-based guideline for the assessment and management of polycystic ovary syndrome. Human Reproduction, 33(9), 1602-1618. https://doi.org/10.1093/humrep/dey256

Turner-McGrievy, G. M., Davidson, C. R., Wingard, E. E., \& Billings, D. L. (2014). Low glycemic index vegan or low-calorie weight loss diets for women with polycystic ovary syndrome: a randomized controlled feasibility study. Nutrition Research, 34(6), 552-558. https://doi.org/10.1016/j.nutres.2014.04.011

Vollmer, W. M., Sacks, F. M., Ard, J., Appel, L. J., Bray, G. A., Simons-Morton, D. G., ... Karanja, N. (2001). Effects of Diet and Sodium Intake on Blood Pressure: Subgroup Analysis of the DASH-Sodium Trial. Annals of Internal Medicine, 135(12), 1019. https://doi.org/10.7326/0003-4819-135-12-200112180-00005

Van Dam, E. W., Roelfsema, F., Veldhuis, J. D., Hogendoorn, S., Westenberg, J., Helmerhorst, F. M., ... Pijl, H. (2004). Retention of estradiol negative feedback relationship to LH predicts ovulation in response to caloric restriction and weight loss in obese patients with polycystic ovary syndrome. American Journal of Physiology-Endocrinology and Metabolism, 286(4). https://doi.org/10.1152/ajpendo.00377.2003

Zeng, X., Xie, Y.-jie, Liu, Y.-ting, Long, S.-lian, \& Mo, Z.-cheng. (2020). Polycystic ovarian syndrome: Correlation between hyperandrogenism, insulin resistance and obesity. Clinica Chimica Acta, 502, 214-221. https://doi.org/10.1016/j.cca.2019.11.003

Zhu, J.-ling, Chen, Z., Feng, W.-jie, Long, S.-lian, \& Mo, Z.-C. (2019). Sex hormone-binding globulin and polycystic ovary syndrome. Clinica Chimica Acta, 499, 142-148. https://doi.org/10.1016/j.cca.2019.09.010

\section{Glossary}

BMI: Body Mass Index

DASH: Dietary Approach to Stop Hypertension

FAI: Free androgen index

HoMA-IR: Homeostatic Model Assessment for Insulin Resistance

Low-GI diet: Low glycemic diet

PCOS: Polycystic ovary syndrome

SHBG: Sex hormone binding globin

\section{Copyright Disclaimer}

Copyright for this article is retained by the author(s), with first publication rights granted to the journal.

This is an open-access article distributed under the terms and conditions of the Creative Commons Attribution license (http://creativecommons.org/licenses/by/4.0/). 